\title{
Conduta frente à lesão por hipoclorito de sódio em terapia endodôntica: um relato de prontuário
}

Management of sodium hypchlorite injury in endodontic therapy: a case report

Conducta para lesiones de hipclorito de sodio en terapia endodontica: um informe de proprietario

\section{Odacir BORRIN ${ }^{1}$}

Renata LICKS $^{2}$

Juliana Andréa Corrêa TRAVESSAS ${ }^{3}$

Rúbia da Rocha VIEIRA

Juliane Pereira BUTZE ${ }^{5}$

${ }^{1}$ Graduando do Curso de Odontologia da FSG Centro Universitário da Serra Gaúcha 95020-472Caxias do Sul-RS, Brasil

${ }^{2}$ Doutora em Odontologia, Professora do Curso de Odontologia da FSG Centro Universitário da Serra Gaúcha 95020-472Caxias do Sul-RS, Brasil

${ }^{3}$ Mestre em Clínica Odontológica - Radiologia, Professora do Curso de Odontologia da FSG Centro Universitário da Serra Gaúcha 95020-472Caxias do Sul-RS, Brasil ${ }^{4}$ Doutora em Patologia Bucal, Especialista em Endodontia, Professora do Curso de Odontologia da Universidade FEEVALE $93525-075$ Novo Hamburgo - RS, Brasil ${ }^{5}$ Doutora em Clínica Odontológica - Periodontia, Professora do Curso de Odontologia da FSG Centro Universitário da Serra Gaúcha 95020-472Caxias do Sul-RS, Brasil

\section{Resumo}

Objetivos: O presente estudo teve como objetivo relatar a análise de um prontuário de paciente submetido ao tratamento de uma queimadura por hipoclorito de sódio em mucosa oral devido à extravasamento de hipoclorito de sódio durante o tratamento endodôntico. Materiais e Métodos: Através da análise de prontuários de pacientes atendidos na disciplina de Estágio Supervisionado do Complexo Odontológico da FSG Centro Universitário entre os anos de 2017 e 2018, selecionou-se um prontuário de um paciente que sofreu queimadura acidental devido ao extravasamento de hipoclorito de sódio. Resultados: O extravasamento de hipoclorito resultou numa lesão por queimadura na lingual do elemento 45. Como medidas, o paciente foi orientado a usar medicação sistêmica, medicamento Ad-Muc ${ }^{\circledR}$ associado a bochechos com clorexidina a $0,12 \%$ e sessões de laserterapia de baixa intensidade. Conclusão: O presente relato destaca os problemas associados ao extravasamento acidental de solução irrigadora à base de hipoclorito de sódio. O adequado diagnóstico, juntamente com a conduta adotada, associando medicação sistêmica, tópica e realização de laserterapia, neste caso, se mostrou efetiva.

Descritores: Endodontia; Hipoclorito de Sódio; Acidentes.

\section{Abstract}

Objectives: The aim of the present study was to report the analysis of a patient's record submitted to the treatment of a sodium hypochlorite burn in the oral mucosa due to the extravasation of sodium hypochlorite during endodontic treatment. Materials and Methods: Through the analysis of medical records of patients attended in the Supervised Internship discipline of the FSG Centro Universitário Dental Complex between 2017 and 2018, we selected a medical record of a patient who suffered an accident due to hypochlorite leakage from sodium. Results: Hypochlorite extravasation resulted in a burn injury to the lingual element 45. As measures, the patient was instructed to use systemic medication, Ad-Muc ${ }^{\circledR}$ drug associated with $0.12 \%$ chlorhexidine mouthwash, and low-intensity laser therapy sessions. Conclusion: This report highlights the problems associated with the accidental leakage of sodium hypochlorite-based irrigation solution. The proper diagnosis, together with the adopted approach, associating systemic and topical medication and laser therapy, in this case, proved to be effective.

Descriptors: Endodontics; Sodium Hypochlorite; Accidents.

\section{Resumen}

Objetivos: El objetivo del presente estudio fue informar el análisis del registro de un paciente sometido al tratamiento de una quemadura por hipoclorito de sodio en la mucosa oral debido a la extravasación de hipoclorito de sodio durante el tratamiento endodóntico. Materiales y métodos: a través del análisis de los registros médicos de los pacientes atendidos en la disciplina de pasantías supervisadas del Complejo Dental Centro Universitario FSG entre 2017 y 2018, seleccionamos un registro médico de un paciente que sufrió un accidente debido a una fuga de hipoclorito de sodio. Resultados: La extravasación de hipoclorito resultó en una lesión por quemaduras en el elemento lingual 45. Como medida, el paciente recibió instrucciones de usar medicamentos sistémicos, medicamentos Ad-Muc ${ }^{\circ}$ asociados con enjuagues bucales con clorhexidina al $0,12 \%$ y sesiones de terapia con láser de baja intensidad. Conclusión: Este informe destaca los problemas asociados con la fuga accidental de la solución de riego a base de hipoclorito de sodio. El diagnóstico adecuado, junto con el enfoque adoptado, que asocia la medicación sistémica y tópica y la terapia con láser, en este caso, demostró ser eficaz.

Descriptores: Endodoncia; Hipoclorito de Sodio; Accidentes.

\section{INTRODUÇÃO}

A irrigação dos canais radiculares é uma etapa de primordial importância no processo de debridamento e desinfecção do sistema de canais radiculares durante o tratamento endodôntico ${ }^{1,2}$.

A solução irrigadora atualmente mais utilizada é o Hipoclorito de Sódio $(\mathrm{NaOCl})$, sendo utilizada pela primeira vez como uma solução antisséptica por Dakin, em 1915, para limpeza e desinfecção das feridas dos soldados da I Guerra Mundial. Posteriormente, seu uso foi difundido a outras áreas, sendo, desde então, o irrigante mais utilizado na desinfecção de canais radiculares devido à sua forte atividade antimicrobiana e à sua capacidade de dissolver tecido orgânico ${ }^{3}$. No entanto, esta solução possui uma desvantagem quanto à sua toxicidade para com tecidos biológicos quando usada inapropriadamente durante a prática clínica, podendo levar a acidentes e consequentes complicações para o paciente ${ }^{1,4}$.

Os acidentes com NaOCL, apesar de pouco frequentes, podem resultar em várias complicações para o paciente. Dentre estes, podemos citar injeção inadvertida de NaOCL no tecido periapical, nos seios maxilares, infiltração através de perfuração lateral da raiz, hipersensibilidade e reação alérgica causadas pela aplicação desta substância ${ }^{5}$. Ainda podemos citar queimaduras químicas, quando a solução de $\mathrm{NaOCL}$ extravasa para os tecidos perirradiculares, gerando desde uma queimadura até a necrose do tecido ${ }^{6}$.

Em caso de intercorrências advindas do uso do hipoclorito, é necessário que o profissional saiba identificar o problema ocorrido, bem como intervir da maneira mais adequada com o intuito de controlar a dor até a remissão completa dos sintomas ${ }^{7,8}$. Para 
isso, o profissional pode lançar mão de algumas medidas terapêuticas em casos de complicações oriundas do uso inadvertido da solução irrigadora, tais como: administração de medicamentos locais e sistêmicos, acompanhamento através de exames clínicos e radiográficos e, mais recentemente introduzido como uma alternativa terapêutica, o uso do laser de baixa intensidade (LBI) ${ }^{1,5}$.

O Laser (Light Amplification by Stimulated Emission of Radiation) tem sido utilizado como modalidade terapêutica em várias condições patológicas, com objetivo de acelerar a cicatrização, promover a regeneração tecidual, diminuir a inflamação e aliviar a dor?. Devido às suas características, o LBI tem mostrado resultados positivos no processo de cicatrização de lesões na mucosa oral, de várias origens ${ }^{10}$. Portanto, o objetivo do presente estudo é, através de um relato de prontuário, descrever um acidente com uso de hipoclorito de sódio durante o tratamento endodôntico, bem como a condução do caso até a solução do mesmo.

\section{CASO CLÍNICO}

A pesquisa iniciou a partir do momento que se obteve aprovação no Comitê de Ética em Pesquisa (CEP) da FSG Centro Universitário (CAAE: 17764519.2.0000.5668 - Parecer: 3.497.847). Para tanto, foram analisados os prontuários de pacientes que estiveram em tratamento endodôntico no complexo odontológico da FSG Centro Universitário dentro das disciplinas de Endodontia e Estágios Supervisionados do Curso de Odontologia da FSG Centro Universitário entre os anos de 2017 e 2018. Como critérios de inclusão foram adotados: seleção de prontuário que possuíssem o correto e completo preenchimento; ocorrência de acidente com hipoclorito de sódio durante o tratamento endodôntico. Os critérios de exclusão foram: prontuários incompletos; prontuários que não apresentavam registros fotográficos, ou com precária quantidade de fotografias; e, casos não concluídos. Após esta análise, apenas um prontuário de um paciente foi selecionado.

O prontuário selecionado foi de um paciente de 56 anos de idade, gênero masculino, sem história médica pregressa e sem histórico de reações alérgicas aos compostos clorados, que se apresentou na clínica do complexo odontológico da FSG Centro Universitário buscando tratamento odontológico. Após avaliação clínica e radiográfica, foi constatada a necessidade de se realizar o tratamento endodôntico do elemento 45.

$\mathrm{Na}$ consulta seguinte, se deu o início do tratamento endodôntico, através da abertura coronária, com isolamento absoluto e sob anestesia do nervo alveolar inferior com lidocaína com vasoconstritor. Foi realizado o acesso ao canal, bem como a exploração do mesmo sob irrigação com $5 \mathrm{ml}$ de NaOCL 1\%, colocado medicação intracanal $\left(\right.$ Otosporin $^{\circledR}$ ) e o dente foi selado com selamento duplo provisório (bola de algodão, Cavitec ${ }^{\circledR}$ - Caithec e Vidrion $F^{\circledR}$ - SS White). Uma semana após, na consulta de retorno, o paciente relatou ter sentido dor no elemento e se apresentava com um leve edema submandibular. Se optou pela reabertura do canal, irrigação com NaOCL $1 \%$ e troca de medicação intracanal (Tricresol Formalina ${ }^{\circledR}$ - Biodinâmica) e selamento do mesmo com cimento de ionômero de vidro (CIV) (Vidrion $\mathrm{F}^{\circledR}$ - SS White). Também foi prescrito ao paciente Amoxicilina 500mg, de $8 \mathrm{em} 8$ horas por sete dias e Paracetamol 750mg, de 6 em 6 horas, enquanto houvesse dor. Nesta consulta, foi observado um quadro clínico sugestivo de perfuração a nível subgengival na distal do elemento 45 .

Na sessão seguinte, o paciente relatou não ter sentido mais dor no elemento dentário $45 \mathrm{e}$ diminuição do edema submandibular, no entanto, o mesmo disse estar sentido dor e sensibilidade na região lingual dos dentes 44 e 45 . Ao exame clínico, foi constatada uma lesão ulcerada na região indicada pelo paciente, sugestivo de queimadura por hipoclorito de sódio (Figura 1), sendo então prescrito Ad-Muc ${ }^{\circledR}$ a ser aplicado na região afetada. Acreditase que tenha ocorrido um extravasamento da solução irrigadora pela perfuração a nível subgengival na distal do elemento em questão, mesmo o paciente não tendo relatado sentir nenhum gosto diferente na cavidade bucal.

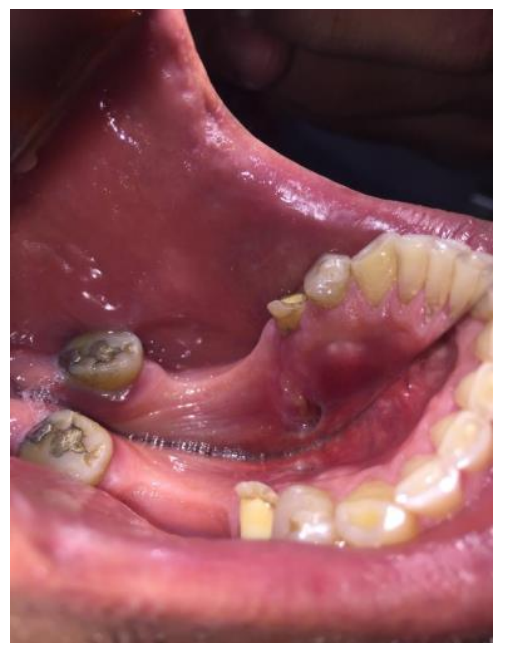

Figura 1: Imagem inicial da lesão.

Nas consultas subsequentes foi observada discreta melhora no quadro clínico do paciente, optando-se, então, pela associação de terapia com LBI. Foram realizadas duas sessões com o laser Therapy EC $\left(\mathrm{DMC}^{\circledR}\right)$, no comprimento de onda de 660 nanômetros (nm) (laser vermelho), dose de aproximadamente $1 \mathrm{~J}$ e fluência de $25 \mathrm{~J} / \mathrm{cm}^{2}$. Como adjuvante a laserterapia foi prescrito solução de digluconato de clorexidina a $0,12 \%$. Após as duas sessões de laserterapia com intervalo de 48 horas, foi 
possível observar regressão da lesão, bem como da sintomatologia dolorosa relatada pelo paciente (Figura 2).

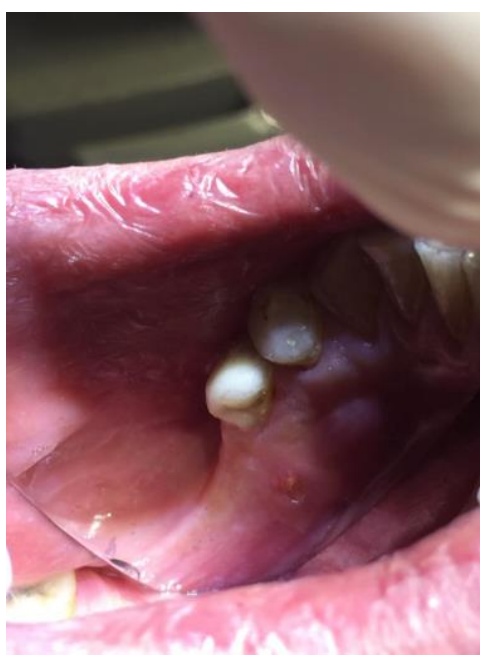

Figura 2: Imagem da regressão da lesão após o tratamento finalizado.

Após a regressão total dos sinais e sintomas, optou-se por prosseguir o tratamento endodôntico, sendo o canal reinstrumentado e irrigado com digluconato de clorexidina $0,12 \%$ até a conclusão do mesmo.

\section{DISCUSSÃO}

O debridamento do sistema de canais radiculares é essencial para sucesso endodôntico, sendo composto por instrumentação mecânica associada ao uso de irrigação antimicrobiana. O hipoclorito de sódio é a solução mais indicada para essa atividade devido ao seu forte poder de assepsia, de remoção de detritos biológicos, dissolver tecido orgânico, forte atividade antimicrobiana e proteolítica, remover produtos microbianos e a camada de smear layer. Com diferentes interações entre substâncias como ácido bórico e bicarbonato de sódio e concentrações variando de 0,5 a $5,25 \%$, não se mostra efetiva com teor abaixo dos $0,3 \%{ }^{6}$. No entanto, o hipoclorito de Sódio se mostra nocivo e tóxico em contato com a mucosa, além de apresentar odor forte e desagradável ${ }^{11}$.

Acidentes com $\mathrm{NaOCl}$ se mostram prejudiciais à saúde. Esta substância em altas concentrações causa danos a tecidos vitais como ulceração, necrose, inibição da migração de neutrófilos, danos às células endoteliais e fibroblásticas ${ }^{6}$. O contato dos tecidos perirradiculares com o $\mathrm{NaCOl}$ pode resultar em reação inflamatória variável, desde reações instantâneas até horas depois da exposição ${ }^{1,5}$. Como protocolo de acidentes com esta solução, em casos mais graves, se deve encaminhar o paciente ao hospital para administração de antibióticos e anti-inflamatórios. Em alguns casos, a associação de esteroides intravenosos e drenagem cirúrgica são necessárias, dependendo da extensão do edema e necrose ${ }^{1}$.
Acidentes com injeção de solução de $\mathrm{NaCOl}$ em tecido gengival e tecidos moles na mucosa oral ocorrem com certa frequência, decorrente da reutilização indevida de anestubos vazios com a solução de hipoclorito de sódio. Complicações diversas podem ocorrer de acordo com a concentração da solução, podendo provocar hematomas, dores e necrose tecidual ${ }^{12}$.

Assim como relatado no presente estudo, em caso de extravasamento durante tratamento endodôntico com hipoclorito com concentração de $1 \%$, o sintoma se mostra de maneira imediata com dor intensa e formação de edema resultando em necrose na mucosa alveolar adjacente. Bem como relatado neste caso, o uso do protocolo convencional (uso de antibiótico, anti-inflamatório e analgésico) conciliando a terapia com LBI para o tratamento é bem indicado 5 .

O uso da terapia com LBI vem se mostrando um grande aliado da Medicina e da Odontologia como tratamento regenerativo, diminuindo o tempo de remodelação e a qualidade do tecido de neoformação. Estudos mostram que os primeiros testes datam de 40 anos atrás, isso indica que seu mecanismo de ação ainda não está completamente esclarecido $^{10,13}$. Contudo, já é observada a estimulação do LBI na atividade celular, essa terapia se mostra possível através da bioestimulação que se atribui ao aumento da atividade celular com a interação do estímulo energético com os tecidos biológicos ${ }^{13}$.

Diferentes tipos de LBI são propostos na Odontologia que fornecem energia de modo contínuo e pulsátil com diferentes comprimentos de onda ${ }^{14}$. Diversas são também as indicações desta terapia pois a mesma é capaz de estimular a osseointegração logo após procedimentos cirúrgicos ${ }^{14,15}$, proporcionando estímulo celular e reparo tecidual de lesões na mucosa oral e tecidos adjacentes após traumas ${ }^{15,16}$.

A melhor maneira de se evitar acidentes durante a irrigação endodôntica com hipoclorito de sódio é adotar medidas preventivas ${ }^{17}$. Para que esse tipo de acidente não ocorra, é essencial que o profissional tenha um conhecimento abrangente da morfologia dentária e da anatomia do sistema de canais radiculares ${ }^{18}$, bem como realize adequadamente os procedimentos de isolamento absoluto durante o atendimento endodôntico.

CONCLUSÃO

O presente relato destaca os problemas associados ao extravasamento acidental de solução irrigadora à base de hipoclorito de sódio. $\mathrm{O}$ adequado diagnóstico, juntamente com a conduta adotada, associando medicação sistêmica, tópica e realização de laserterapia, neste caso, se mostrou efetiva. 


\section{REFERÊNCIAS}

1. Noites R, Carvalho MF, Vaz IP. Complicações que podem surgir durante o uso do hipoclorito de sódio no tratamento endodôntico. Rev Port Estomatol Cir Maxilofac. 2009; 50(1):53-56.

2. Salum G, Barros Filho S, Rangel LFGO, Rosa RH, dos Santos SSF, Leão MVP. Hipersensibilidade ao hipoclorito de sódio em intervenções endodônticas. Rev Odontol Univ São Paulo. 2012;24(3):200-8.

3. Borin G, Becker AN, Oliveira EPM. A história do hipoclorito de sódio e a sua importância como substância auxiliar no preparo químico mecânico de canais radiculares. Rev Endod Pesq Ensino On Line. 2007;3(5):1-5.

4. Witton R, Brennan PA. Severe tissue damage and neurologic defict following extravasation of sodium hypochlorite solution during routine endodontic treatment. $\mathrm{Br}$ Dent J. 2005; 198(12):749-50.

5. Bramante CM, Duque JA, Cavenago BC, Vivan RR, Bramante AS, Andrade FB, Duarte MAH. Use of a 660-nm laser to aid in the healing of necrotic alveolar mucosa caused by extruded sodium hypochlorite: a case report. J Endod. 2015;41(11):1899-902.

6. Tenore G, Palaia G, Ciolfi C, Mohsen M, Battisti A, Romeu U. Subcutaneous emphysema during root canal therapy: endodontic accident by sodium hypoclorite. Ann Stomatol (Roma). 2017; 8(3):117-122.

7. Hulsmann M, Hahn W. Complications during root canal irrigation - literature review and case reports. Int Endod J. 2000;33(3):186-93.

8. Silva JPM, Boijink D. Acidente com hipoclorito de sódio durante tratamento endodôntico: Análise de prontuário. Rev Odontol Araçatuba. 2019;40(1):25-8.

9. Ribeiro MS, Silva DFT, Zezell DM, Nunez SC. Laser de baixa intensidade. A Odontologia e o laser. São Paulo: Quintessense; 2004.

10. Azzi VJB, Di Pietro SN. Aplicação da laserterapia no tratamento de queimaduras: uma revisão sistemática. Rev Bras Terap e Saúde. 2012;3(1):15-26.

11. Graça BP. O Hipoclorito de sódio em Endodontia [dissertação]. Porto: Faculdade de Ciências da Saúde/Universidade Fernando Pessoa; 2014.

12. Gursoy UK, Bostanci V, Kosger HH. Palatal mucosa necrosis because of accidental sodium hypochlorite injection instead of anaesthetic solution. Int Endod J. 2006;39(2):157-61.

13. Freitas RPA, Barcelos APM, Nóbrega BM, Macedo AB, Oliveira AR, Ramos AMO et al. Laserterapia e microcorrente na cicatrização de queimadura em ratos: terapias associadas ou isoladas?. Fisioter Pesqui. 2013;20(1):24-30.
14. Prockt AP, Takahashi A, Pagnoncelli RM. Uso de terapia com laser de baixa intensidade na cirurgia bucomaxilofacial. Rev Port Estomatol Cir Maxilofac. 2008;49(4):247-55.

15. Noba C, Mello-Moura ACV, Gimenez T, Tedesco TK, Moura-Netto C. Laser for bone healing after oral surgery: systematic review. Lasers med Sci. 2018;33(3):667-74.

16. Rocha Júnior AM, Oliveira RG, Farias RE, Andrade LCF, Aarestrup FM. Modulação da proliferação fibroblástica e da resposta inflamatória pela terapia a laser de baixa intensidade no processo de reparo tecidual. An Bras Dermatol. 2006;81(2):150-56.

17. Soares RG, Dagnese C, Irala LED, Salles AA, Limongi O. Injeção acidental de hipoclorito de sódio na região periapical durante tratamento endodôntico: relato de caso. RSBO. 2006; 4(1):17-21.

18. Doherty MAH, Thomas MBM, Dummer PMH. Sodium hypochlorite accident - a complication of poor access cavity design. Dent Update. 2009;36(1):7-12.

\section{CONFLITO DE INTERESSES}

Os autores declaram não haver conflitos de interesse.

\section{AUTOR PARA CORRESPONDÊNCIA}

\section{Juliane Pereira Butze}

juliane.butze@fsg.edu.br

Submetido em 11/11/2019

Aceito em 23/06/2020 